\title{
The Form of the Benardete Dichotomy
}

\section{Nicholas Shackel}

\begin{abstract}
Benardete presents a version of Zeno's dichotomy in which an infinite sequence of gods each intends to raise a barrier iff a traveller reaches where they intend to raise their barrier. In this paper I exhibit the abstract form of the Benardete Dichotomy. I show that the diagnosis based on that form can do philosophical work not done by earlier papers rejecting Priest's version of the Benardete Dichotomy, and that the diagnosis extends to a paradox not normally classified as a dichotomy. I show how the form is exploited to generate paradox.
\end{abstract}

1 Introduction

2 The form of the Benardete dichotomy

3 Applying the unsatisfiable pair diagnosis

4 Exploiting the form

\section{Introduction}

Benardete ([1964], pp. 259-60) presents a version of Zeno's dichotomy in which an infinite sequence of gods each intends to raise a barrier iff a traveller reaches where they intend to raise their barrier. Priest presents a formalisation which replaces intentions of gods to raise barriers with a demon who mines the line in such a way that 'passing certain spots brings barriers spontaneously into existence ([1999], p. 2). In refuting Priest, Yablo presents an infinite set of demons each of whom will say 'YES if and only if all the earlier-calling demons have called NO' ([2000], p. 150). Perez Laraudogoitia] ([2003]) offers a variant of Benardete’s dichotomy which he claims is not refuted by his own earlier ([2000]) refutation of Priest. Hawthorne ([2000]) and Angel ([2001]) exploit variants which appear not to be refuted by the earlier papers. There is a common contradictory form underlying all of these dichotomies. That form is also embedded in some other paradoxes, including Yablo's ([1993]) paradox without self reference.

In the second section I show what the form of the Benardete Dichotomy is. In the third section I show that the diagnosis based on the form can do philosophical work not done by Yablo ([2000]) and Perez Laraudogoitia ([2000]). In the final section I show how the form is exploited to generate paradox in the Benardete Dichotomy.

\section{The form of the Benardete dichotomy}

For the purposes of this discussion, let $x$ range over an infinite set $\mathrm{S}$ linearly ordered by a relation called 'before' $(\ldots<<\ldots)$. Members of $\mathrm{S}$ may represent or be times or places on a line. Let such sets which have no first member be called unbegun sets, and the condition of having no first member be called the unbegun condition ( $\forall x \exists y y<<$ $x) .{ }^{1}$ Let such sets which have a first member be called begun sets and let $b$ be their

\footnotetext{
${ }^{1}$ Examples of unbegun sets are the positive reals, $\mathbb{R}^{+}$, the sets $\left\{1 / 2^{n}\right\}$ and $\{1 / n\}$ (i.e. the sets $\{x \in \mathbb{R}: x>0\},\left\{x \in \mathbb{R}: x=1 / 2^{n}\right.$ for $\left.n \in \mathbb{N}\right\}$ and $\{x \in \mathbb{R}: x=1 / n$ for $\left.n \in \mathbb{N}\}\right)$, under their usual ordering.
} 
first member. Let ' $E$ ' be a predicate symbol, whose interpretation is intended to be a property of being instantiated at times and places, such as had by events, particles, barriers etc. being at times and places. We have a pair of related predicates: ' $E$ at ...' and ' $E$ before ...'. In general, to be before is ambiguous between being somewhere before and being at all before, so we define ' $E$ somewhere before $\mathrm{x}$ ' to mean $\exists y$ $(y<<x$ \& Ey), 'E all before $x$ ' to mean $\forall y(y<<x \rightarrow$ E $y)$ and 'E nowhere before $x$ ' to mean $\neg \exists y(y<<x$ \& Ey).

The Benardete dichotomies apply the seemingly innocuous condition that something happens at a place or time iff it does not happen anywhere before that place or time. We might call this the 'At iff Nowhere Before condition':

Condition ANB: $\quad$ For all $x$ in S, E at $x$ iff E nowhere before $x$.

Condition ANB is not appealed to directly. I claim that the indirection is not innocent but, as we shall eventually see, guileful. Benardete says

A man decides to walk one mile from A to B. A god waits in readiness to throw up a wall blocking the man's further advance when the man has travelled $1 / 2$ a mile. A second god (unknown to the first) waits in readiness to throw up a wall of his own blocking the man's further advance when the man has travelled $1 / 4$ mile. A third god ... etc. ad infinitum. (Benardete [1964], pp. 259-60).

The set $S$ is the unbegun set of places $\left\{1 / 2^{n}\right\}$. Implicit is that the $n$th god doesn't put up his wall unless the man reaches $1 / 2^{n}$. So

1. for all $x$ in $\mathrm{S}$, the man reaches $x$ iff a god puts up a wall at $x$. Also implicit is 'that the man does not stop unless a barrier is put in his way'(Yablo [2000], p.148). So the man will reach $x$ if there was no barrier before $x$ i.e. no god put up a wall before $x$. Likewise, if the man reaches $x$ there was no barrier before $x$ i.e. no god put up a wall before $x$. So

2. for all $x$ in S, the man reaches $x$ iff a god puts up a wall nowhere before $x$. Putting 1 and 2 together we see we have condition ANB embedded in Benardete's dichotomy:

3. for all $x$ in S, a god puts up a wall at $x$ iff a god puts up a wall nowhere before $x$.

Consider Priest's premisses for his version of the dichotomy:

(2) $\mathrm{B} x \& x<y \rightarrow \sim \mathrm{R} y^{2}$

(3) $\sim \exists x(x<y \& B x) \rightarrow R y$

(4) $x \leq 0 \rightarrow \sim B x$

(5) $x>0 \rightarrow(B x \leftrightarrow R x / 2)$ (Priest [1999], p.2)

Sentence (5) says that there is a barrier at $x$ iff you reach $x / 2$. The effect of sentences (2) and (3) is just that you will reach $y$ iff there is no barrier before $y .{ }^{3}$ Put them

\footnotetext{
${ }^{2}$ I've swapped variables from the original to make it easier to follow.

${ }^{3}$ The antecedent of (2) is implicitly existentially quantified, $\exists x(\mathrm{~B} x \& x<y) \rightarrow \sim \mathrm{R} y$, so by contraposition, Ry $\rightarrow \sim \exists x(x<y \& B x)$, which with (3) gives Ry $\leftrightarrow \sim \exists x(x<y \& B x)$.
} 
together, and you get a barrier at $x$ iff there is no barrier before $x / 2$. Perez Laraudogoitia derives the same result formally from the same three sentences in his derivation of his sentence (10):

(10) $\forall x(x>0 \rightarrow(B x \leftrightarrow \sim \exists y(y<x / 2 \& B y)))$ (Perez Laraudogoitia [2000], p. 154)

Nothing turns on the minor modification incumbent on the distinction between 'before $x$ ' and 'before $x / 2$ ' just because the $x$ is ranging over an unbegun set in which $x / 2$ is always before $x$. Priest could just as well have used ' $x$ ' instead of ' $x / 2$ ' in (5).

The set $\mathrm{S}$ is the unbegun set, $\mathbb{R}^{+}$, and condition ANB, for all $x$ in $\mathrm{S}$, there is a barrier at $x$ iff there is a barrier nowhere before $x$. So Priest's sentences (2) (3) and (5) effectively disguise an example of condition ANB.

Likewise, we can see that Yablo's demons, in attempting to say 'YES if and only if all the earlier-calling demons has called NO' (Yablo [2000], p.150), are attempting to conform to condition ANB. The set $S$ is the unbegun set of times $\left\{1 / 2^{n}\right\}$. All earlier calling demons calling No is logically equivalent to Yes called nowhere before. So we have condition ANB, for all $t$ in S, a demon calls YES at time $t$ iff a demon calls YES at no time before $t$.

Condition ANB has a surprising consequence for unbegun sets. Let $\mathrm{S}$ be an unbegun set, and $y$ be any member of S before $x(y<<x)$. Then for all $x$ in $S$ and for all $y$ before $x$

1) $\quad \mathrm{E}$ at $x$ iff $\mathrm{E}$ nowhere before $x$ (condition ANB)

which is equivalent to

2) not $\mathrm{E}$ at $x$ iff $\mathrm{E}$ somewhere before $x$

By substitution in 2) we get

3) not $E$ at $y$ iff $E$ somewhere before $y$

Since $y$ ranges over all members of $S$ which are before $x$ we have

4) E nowhere before $x$ iff not $\mathrm{E}$ at $y$

so from the chain of biconditionals (1), (4) and (3) (in that order) we have

5) $\quad \mathrm{E}$ at $x$ iff E somewhere before $y$

Clearly, since $y$ is before $x$,

6) E somewhere before $y$ only if E somewhere before $x$

so from (5) and (6)

7) $\quad$ E at $x$ only if E somewhere before $x .{ }^{4}$

hence from (7) and (2)

8) $\quad \mathrm{E}$ at $x$ only if not $\mathrm{E}$ at $x$

whence

9) $\operatorname{not} \mathrm{E}$ at $x$

\footnotetext{
${ }^{4}$ Comparing lines 1) and 7) shows a contradiction to be lurking.
} 
So the seemingly innocuous condition ANB, which apparently permits $\mathrm{E}$ at $x$ under an acceptable bicondition, imposes the impossibility of $\mathrm{E}$ at $x$ when $x$ ranges over an unbegun set.

A few additional steps gives us an explicit contradiction.

10) E somewhere before $x \quad 2,9$

11) E somewhere before $x$ iff $\exists z$ before $x$ and $E$ at $z$

12) E at $a \quad 10,11$, unbegun condition, EI

13) not $\mathrm{E}$ at $a$

14) $\quad \mathrm{E}$ at $a$ and $\quad$ Not $\mathrm{E}$ at $a \quad 2,13$

So the application of condition ANB to an unbegun set is contradictory.

Condition ANB is satisfiable by a begun set, and forces $\mathrm{E}$ at $b$ ( $b$ is defined to be the first member), for consider the RHS of condition ANB with $b$ substituted for $x$ : E nowhere before $b$. In full, this reads ' $\forall y y<<b \rightarrow \neg \mathrm{E} y$ '. Since $b$ is the first member of a begun set $y<<b$ is false for all $y$ and therefore ' $\forall y y<<b \rightarrow \neg E y$ ' is true of begun sets. ${ }^{5}$ Hence by condition ANB, E at $b$. Whence by condition ANB, not E at $x$ for all $x$ after $b .^{6}$

The path to the contradiction is blocked for begun sets. The step to line (3) would be invalid because of the restriction on $y$ being a member of $S$ before $x$. Only unbegun sets promise such a member for all $x$ in S. The detail of (3)'s derivation goes

2) not $\mathrm{E}$ at $x$ iff E somewhere before $x$

Now since (2) applies to all $x$ in S, if there is a $y$ before $x$, it will apply to that $y$. So we have

2b) if there is a $y$ before $x$ then (not $\mathrm{E}$ at $y$ iff $\mathrm{E}$ somewhere before $y$ )

From which we get, by the unbegun condition, $\forall x \exists y y<<x$, and detachment

3) not $\mathrm{E}$ at $y$ iff $\mathrm{E}$ somewhere before $y$

So although (2) and (2b) are true of begun sets, we cannot get (3) for begun sets because we need the unbegun condition for the detachment. Obviously (3) is false for the first member of begun sets, $b$, since it says not $\mathrm{E}$ at $b$ iff there exists a $z$ before $b$ such that $\mathrm{E}$ at $z$, but since $b$ is before all other members of $\mathrm{S}$ there can be no such $z$.

The step to line (12) would also be invalid because it uses the unbegun condition.

\footnotetext{
${ }^{5}$ Whereas for unbegun sets, because for all $x$ in $S$ there exists a $y$ such that $y<<x$, there is no c in S such that for all $x, x<<\mathrm{C}$ is false, and therefore the RHS of B need not be true for any member of S.

${ }^{6}$ Robert Black has pointed out to me that this fact can be shown very shortly as follows: Let $\mathrm{R}$ be any connected relation on $\mathrm{S}$. Then if condition ANB holds for some property $\mathrm{E}$ it can be shown to hold for only one member of $S$ and that member will bear $R$ to every other member of $\mathrm{S}$. Whence if the connected relation is an ordering, thus transitive, the unique $\mathrm{E}$ must be the first member, so the set $\mathrm{S}$ must be begun.
} 


\section{The unsatisfiable pair diagnosis}

So now we have the form of the Benardete dichotomy: Embedded in it is a pair of conditions which are jointly unsatisfiable:

1. Condition ANB: For all $x$ in S, E at $x$ iff E nowhere before $x$.

2. The set $S$ in condition ANB is an unbegun set.

Call this the unsatisfiable pair diagnosis. Benardete predicates the execution of intentions on the non-execution of prior intentions where there is no first time or place for action or inaction. Priest predicates the existence of barriers on the absence of prior barriers with no first place for a barrier. Yablo predicates demons saying yes on no previous demons saying yes with no first time for a demon to speak. Each is a matter of applying condition ANB where E respectively is the execution of prior intentions, the existence of a barrier, the demon speaking yes, whilst $x$ respectively ranges over an unbegun set of places, places, and times. Therefore, by so predicating they did not allow any such executions, barriers or speaking to happen under a condition, but made them contradictory a priori.

\section{Applying the unsatisfiable pair diagnosis}

Perez Laraudogoitia and Yablo both offer diagnoses of the Benardete dichotomy which focus on Priest's version. Perez Laraudogoitia ([2000]) derives a formal contradiction from Priest's premisses. Yablo discusses the coherence of the infinitude of intentions of Priest's demons who "“mine” an area of space in accordance with [premisses] (4) and (5)' (Priest [1999], p. 2) by discussing the coherence of an analogous infinitude of demons, and concludes that 'the gauntlet of demons.... is saved from incoherence only by the assumption that $\alpha$ [the travelling particle in Priest's version] stops at zero' ([2000], p. 150).

The unsatisfiable pair diagnosis is compatible with the latter diagnoses, but is more general and for that reason can do more philosophical work. First, it applies to a variant of Benardete's dichotomy proposed by Perez Laraudogoitia which he says is not resolved by either his earlier paper or by Yablo's diagnosis (Perez Laraudogoitia [2003], pp. 130-1). Second, Hawthorne ([2000]) and Angel ([2001]) use variants of Benardete's and Zeno's dichotomies to motivate the rejection of certain plausible principles. In neither case is it evident that Perez Laraudogoitia and Yablo's earlier papers can show that those arguments fail, let alone explain why they do. The unsatisfiable pair diagnosis, however, illuminates Hawthorne's and Angel's uses of dichotomies and thereby undermines what they take themselves to have achieved. Thirdly, the unsatisfiable pair diagnosis applies to a paradox not normally classified with the dichotomies, namely, Yablo’s paradox without self-reference ([1993]). Finally, it lays bare a general mechanism for constructing these paradoxes. ${ }^{7}$

\section{Perez Laraudogoitia}

Perez Laraudogoitia claims his new variants are not refuted by his or Yablo’s earlier paper partly because

\footnotetext{
${ }^{7}$ There may be an argument to be had turning on whether when a paradox uses 'for all natural numbers...' we are being offered a set of sentences in a formal language specified in a metalanguage, and hence on whether we need to address the relation of $\omega$-inconsistency to inconsistency and determine whether implicit appeals are being made to $\omega$-rule inferences. For the sake of space, I shall not be addressing those arguments.
} 
from the fact that the particle can only interact with the barriers, and that therefore only the barriers can stop it, it does not follow that if it does not interact with any barrier it will not stop. This latter is in fact what Priest (1999, p. 2 axiom (3)) explicitly assumed, trivially turning Benardete's paradox into a contradiction. ... What this paradox shows is that the particle can interact with the set of barriers... without interacting with any one barrier...[failing to] recognise this fact...commits a division fallacy. (Perez Laraudogoitia [2003], p. 126)

This point makes sense when there are barriers in place, but is more difficult to understand if the barriers are non-existent. In Benardete's dichotomy the man is stopped even though no god creates any barrier. Perez Laraudogoitia's remark seems to imply the man is stopped by interacting with the set of non-existent barriers and that to derive the contradiction requires committing the division fallacy of thinking that in order to interact with a set of non-existent barriers one must interact with one of the non-existent barriers. But that doesn't seem to be the problem at all. Prima facie, one would have thought the problem is being stopped by non-existent barriers at all, not by how many non-existent barriers get in on the act. Nevertheless, if we grant Perez Laraudogoitia his point with respect to non-existent barriers then his claim that it is not resolved by his or Yablo's earlier paper is plausible. I shall now show that the condition placed on the gods in Perez Laraudogoitia's new variants is in each case condition ANB and that the set $\mathrm{S}$ in each case is unbegun.

I will construct a temporal version of the paradox of the gods. Now the particle is moving with constant velocity $\mathrm{v}$ for $\mathrm{t}<0$. A god decides to stop it at $t=1 / 2$, wherever it is, if it is not at rest already at that instant. A second god (unknown to the first) decides to stop it at $t=1 / 3$, wherever it is, if it is not at rest already at that instant. A third god... and so on ad infinitum. (Perez Laraudogoitia [2003], p. 124)

Here the set $\mathrm{S}$ is the set of times $\{t=1 / n\}$. Condition ANB is that for all $t$ in $\mathrm{S}$, a god stops the particle at time $t$ iff no god stop the particle before $t$. The $L$ to $R$ direction holds because if a god stops the particle at $t$ it must have been in motion prior to $t$ and so no gods stopped it before $t$. The $R$ to $L$ direction holds because unless the gods stop it before $t$ it will not be at rest, so a god will stop it at $t$. These remarks hold for his other variants. Perez Laraudogoitia's next variant avoids the problem of 'infinite force at one instant' ([2003], p. 127).

In general, God- $n$ decides to act as follows: wherever the particle is at $\mathrm{t}$ $=1 /(n+1)$, he will take it to rest, if it is not at rest already, in the time interval [1/(n+1), 1/n]. (Perez Laraudogoitia [2003], p. 127)

Here the set $S$ is the set $\{\mathfrak{I} \subseteq \mathbb{R}: \mathfrak{I}=[1 /(n+1), 1 / n]\}$. Condition ANB is that for all intervals of time $\mathfrak{I}$ in $\mathrm{S}$, a god will stop the particle during interval $\mathfrak{I}$ iff a god stops the particle during no interval before $\mathfrak{I}$. Perez Laraudogoitia's final variant is essentially the same as the latter, only allowing that perhaps some physical processes were instant ('class $C$ - processes'), whilst confining the gods to using processes which require intervals of time ('class $C$ processes').

let God- $n$ act like this: wherever the particle is at $t=1 /(n+1)$, it will take it to rest by some class $C$ process in the time interval $[1 /(n+1), 1 / n]$, if it 
is not at rest [already], or if it is at rest but got there by some class $C^{-}$ process. (Perez Laraudogoitia [2003], p. 128)

Here the set $S$ is the set $\{\mathfrak{I} \subseteq \mathbb{R}: \mathfrak{I}=[1 /(n+1), 1 / n]\}$. Condition ANB is that for all intervals of time $\mathfrak{I}$ in $\mathrm{S}$, a god will stop the particle during interval $\mathfrak{I}$ by a class $C$ process iff a god stops the particle during no interval before $\mathfrak{I}$ by a class $C$ process.

\section{Hawthorne}

We turn to a paradox for which the division fallacy point is more clearly true. I place impenetrable barriers of appropriately diminishing thicknesses at each of $\{1 / n\} ;^{8}$ a sphere travelling from the left couldn't pass 0 without passing through infinitely many barriers, yet if it stopped at 0 it would do so without being stopped by any particular barrier. This sounds odd, but is not contradictory without the assumption Perez Laraudogoitia criticised. If we think otherwise, it is because we think that what Hawthorne calls the contact principle is generally true:

The contact principle: If $y$ is the fusion of $x$ 's and $z$ contacts $y$, then $z$ contacts one of the $x$ 's. ([2000], p. 626)

To think that the contact principle applies to infinitely many barriers is to commit the division fallacy: one can be stopped by a fusion of infinitely many barriers without being stopped by any single barrier. Hawthorne claims that this diagnosis (rejecting the contact principle for infinite fusions) resolves the two dichotomies Benardete gives before his dichotomy of the gods.

Let the peal of a gong be heard in the last half of a minute, a second peal in the preceding $1 / 4$ minute... etc. ad infinitum. ... Let us assume that each peal is so very loud that, upon hearing it, anyone is struck deaftotally and permanently. At the end of the minute we shall be completely deaf (any one peal being sufficient), but we shall not have heard a single peal! .... We are now tempted to coin the barbarous neologism of a before effect.

A man is shot through the heart during the last half of a minute by A. B shoots him through the heart during the preceding $1 / 4$ minute ...etc. ad infinitum. Assuming that each shot kills instantly (if the man were alive), the man must be already dead before each shot. (Benardete [1964], p. 255-59)

Hawthorne says that what deafens is the fusion of sound waves, what kills is the fusion of bullets, and that only our adherence to the contact principle leads us to find these before effects contradictory. I shall offer a shooting case which Hawthorne's rejection of the contact principle does not resolve, and then show that the unsatisfiable pair diagnosis applies to it.

The plausibility in the case of the fusion of walls rests on the topology of regions of space and what should be counted as contact when objects may occupy open, half open, or closed regions. ${ }^{9}$ A definition of contact which will cover all the possible combinations Hawthorne considers on page 626 is this: contact occurs iff the

\footnotetext{
${ }^{8}$ Thicknesses of $1 / 2^{n}$ would do.

${ }^{9}$ I make use of the standard topological definitions. See, for example, Sutherland [1998].
} 
boundaries of the regions occupied by the objects share at least one point. ${ }^{10}$ Now the fusion of walls occupies a region open to the left and its boundary on the left is a vertical plane through the origin, $O,{ }^{11}$ so there is a perfectly good sense in which the sphere (whatever sort of region of space it occupies) contacts the fusion when its boundary shares the point $O$ with the left hand boundary of the fusion. ${ }^{12}$

It is not obvious that there is, and Hawthorne does not provide, a similar spelling out of what is happening in the cases of the gong or the shooting. It may be possible to do so in the gong case, provided the gong stays still, otherwise it might fall to the objection I am about to make about the shooting case.

In the shooting case, perhaps what Hawthorne has in mind is all the bullets travelling the same path to the man's heart, so that the fusion of bullets occupies a region of space with similar properties to that occupied by the region of barriers. Analogously to the boundary of the fusion of walls approaching the sphere (if we set our inertial frame on the sphere), we have a picture of the boundary of the fusion of bullets travelling so as to contact the victim's heart just as each bullet would, only contacting it at time zero and so killing him before any actual bullet does. ${ }^{13}$ Hence the before effect of the fusion of shooters.

Grant for now the plausibility of that account. It will not suffice. Consider instead the infinitude of shooters distributed around the victim so that the last shooter shoots from the north and the bearing from north of the shooter who shoots during the time interval $\left[1 / 2^{n+1}, 1 / 2^{n}\right]$ is one radian greater than the shooter who shoots during $\left[1 / 2^{n}\right.$, $\left.1 / 2^{n-1}\right]$. Since there are $2 \pi$ radians in a circle and $\pi$ is an irrational number there is no common path for the bullets. ${ }^{14}$ I can stipulate that the bullet sizes decrease appropriately to avoid overlapping paths at the surface of the heart. ${ }^{15}$ When we now enquire about the boundary of the fusion of these bullets, and in particular about that part of the boundary which is going to do the killing, the analogy collapses. For in the wall case the vertical plane running through 0 is a boundary of the fusion because (to

\footnotetext{
${ }^{10}$ The boundary of a region $R$ in a topological space $T$ is $\mathrm{Cl}(R) \cap \mathrm{Cl}(T-R) . \mathrm{Cl}(R)$ is the closure of $R$, which is the union of $R$ with all its limit points. Objects are impenetrable so we don't need to have a definition which covers engulfment.

${ }^{11}$ The boundary is part of the $y$-z plane. I take the $x$-axis to be horizontal and $y$-axis to be vertical. I won't burden the reader with three dimensional coordinates.

${ }^{12}$ An alternative not considered by Hawthorne and avoiding the use of fusions is to use impenetrability. We can define penetration as follows:
}

Object A penetrates D iff there exists a point $x$ on the boundary of the space occupied by A for which there exists an open ball $B(x)$ such that $B(x)$ is entirely contained in the region of space occupied by $D$.

Then rather than having to appeal to contact to explain the power of a barrier to stop an object, we appeal to impenetrability. The ball stops at zero because penetration is impossible. If it passes zero there will be at least one wall (in fact infinitely many) which it penetrates, but penetration is impossible.

${ }^{13}$ Hawthorne supposes 'if a metal object penetrates $1 / 4$ inch into the heart, then the person dies at that very moment' ([2000], p. 627) but we can just as well suppose death is instantaneous on contact.

${ }^{14}$ Because for two bullets to share a path would require two shooters to have the same bearing, which would required there to be $k, m \in \mathbb{N}$ for which $2 k \pi=m$. But that contradicts the irrationality of $\pi$.

${ }^{15}$ Or be point sized, if necessary. 
put it very loosely) the walls 'tend to that plane in the limit'. But in the bullet case there is no such 'limiting' boundary to hit the heart at time zero. By construction, I have eliminated that 'limiting' boundary and so the boundary of the fusion is no more than the union of all the boundaries of all the individual bullets. None of those boundaries contacts the heart at time zero, so the boundary of the fusion can't do the killing at time zero. In the wall case the paradox of the before effect is accounted for by rejecting the contact principle and accepting that the sphere contacts the fusion without contacting any individual wall by contacting the boundary of the fusion at 0 . That explanation fails in the bullet case. In the bullet case we can reject the contact principle and accept that the heart is contacted if contacted by the boundary of the fusion of bullets even if contacted by no individual bullet. But the boundary of the fusion of bullets is not in contact with the heart at time zero. Yet there is no time after time zero at which he can be alive. So the paradox remains unexplained by a rejection of the contact principle.

Now I have had to speak somewhat loosely in order to keep this objection both comprehensible and short. I think it is evidently provable, but it is a non-trivial mathematical task to prove it. At the very least, I have made it evident that Hawthorne's picture leaves it quite unclear whether it generalises to the shooting case. If it doesn't then the diagnosis by falsity of the contact principle does not apply in that case.

My diagnosis, however, does apply. In the shooting case, the set $\mathrm{S}$ is the set of time intervals $\left\{\mathfrak{I} \subseteq \mathbb{R}: \mathfrak{I}=\left[1 / 2^{n+1}, 1 / 2^{n}\right], n \in \mathbb{N}\right\}$, which set is unbegun, and condition ANB is that for all $\mathfrak{I}$ in $S$, a man kills the victim during $\mathfrak{I}$ iff a man kills the victim during no interval before $\mathfrak{I}$.

Hawthorne concedes that falsity of the contact principle does not solve the Benardete dichotomy because 'after all, in that case there is not a wall thrown down and so there is no fusion of walls with which to come into contact' ([2000], p. 627). Hawthorne would therefore seem to agree with me that Perez Laraudogoitia's remarks about the division fallacy fall short of the mark. In place of Benardete's dichotomy, Hawthorne considers a variant in which infinitely many assassins are each disposed to kill Bob during a time interval should he be alive at the beginning of the time interval (see [2000], pp. 627-30). Hawthorne concludes

The puzzlement resides in the fact that we think of the assassins as individually having to do something...yet... the fusion [of assassins] causally secures the assassination of Bob without even moving! Nor does the fusion need to undergo any other type of change at all in order to assassinate Bob. Our puzzlement thus relies, I suggest, on our tacit endorsement of the following principle relating fusions to their parts, which we can call the "Change Principle"

If $x$ is the fusion of $y$ 's and $y$ 's are individually capable only of producing effect $e$ by undergoing change, then $x$ cannot, (without the addition of some non-supervening causal power), produce effect $c$ without undergoing change.

This principle is mistaken, which I suggest is a big metaphysical surprise. By suitably combining things that need to change in order to 
produce a result, we can generate a fusion that can produce that result without undergoing change....

The Contact Principle, in full generality, could be given up fairly readily on reflection. The Change Principle has a rather deeper hold on us. ([2000], p. 630)

I think the relevant analogous principles summarised by the Change Principle are evident; for example, in Benardete's dichotomy it would be more naturally expressed in terms of heavenly agents and their actions. We could also omit the fusion and express this in terms amenable to Perez Laraudogoitia's criticism of division fallacies.

I think Hawthorne's position is incoherent. It is worth pointing out a difference between the case of the Contact Principle and that of the Change Principle. In the former case, we have an account of contact to replace the contact principle, namely that one can contact a fusion by contacting its boundary. The plausibility of this account rests on an independent theoretical concern: the necessity for an account of contact between open objects (objects occupying open regions of space), which can only happen if two objects can be in contact by sharing a boundary point, even though neither occupies its boundary. In the case of the Change Principle, the main reason for rejection would seem to be avoiding the puzzlement of Benardete's dichotomy. I don't see, as I do in the case of the Contact Principle, independent theoretical concerns which when applied make sense of why the principle should be given up. On the contrary, I would think our independent theoretical concerns give substantial support to the Change Principle. When Hawthorne says

getting together enough of the mundane things and suitably arranging them is all by itself logically sufficient to entail changeless causation [the absence of change is in the thing doing the causing] ([2000], p. 630).

to me he has offered a reductio. I suspect that changeless causation is an incoherent notion. If they don't have to undergo any change to collectively kill Bob need they even exist in order to kill him? I can arrange for each assassin to be killed at the time he would detect and kill Bob iff Bob is already dead at that time. If we grant that the Change principle is false and that consequently the mere arrangement of assassins must be allowed to be 'by itself logically sufficient', Bob cannot be alive at any time after 1.00p.m. ${ }^{16}$ and so every assassin who would kill Bob has gone out of existence before he would kill him. We would now seem to have to reject yet another principle:

If $x$ is a fusion of $y$ 's, and each $y$ is disposed to cause an effect but ceases to exist before it causes that effect, $x$ does not cause that effect.

Rejecting that principle is pretty wild, since to do so seems to be a matter of accepting causeless causation.

Hawthorne carefully avoids stipulating 'that the laws of nature are such that Bob will survive unless he is [killed] by an assassin' because 'There is no possible world satisfying that description and so the question as to what happens in such a world is illegitimate' ([2000], p. 630). I agree. But he does not avoid impossibility so simply. His survival is supposed to be analogous to the continued motion of the man in Benardete's dichotomy. If we are to permit uncaused deaths for Bob we could just as

${ }^{16}$ His state at 1.00p.m. is undetermined. 
well permit uncaused stops for the man in Benardete's dichotomy, and the whole problem can be forgotten. Hawthorne doesn't say Bob's death is uncaused but that he is killed by the fusion of assassins. He is committed to this on pain of losing his challenge to the Change Principle. So Hawthorne must concede that Bob will survive unless he is killed by something. Given only that, we can find the unsatisfiable pair in his set up without this stipulation, and that, I think, is the source of the incoherence here.

Hawthorne reconstrues his assassins and sets up the situation in a complicated manner, which I will lay out and then simplify in order to locate the unsatisfiable pair.

Make the assassins A-type point particles. Make Bob a B-type point particle....Assassination is the transformation of a $\mathrm{B}$ particle into an $\mathrm{A}$ particle. This occurs by an A particle interpenetrating a $\mathrm{B}$ particle at $\mathrm{t}$ and at that time irradiating $\mathrm{x}$ radiation - this being what constitutes "attack". "Survival" here and in what follows is "remaining as a Bparticle". The causal laws and state of the world are such as to make the following three claims true:

Particle 1 will not move with respect to the point occupied by Bob before 2 p.m.

If at 2 PM Bob still exists as a B particle, then by 2.30, Bob will have been assassinated by particle 1 .

If at 2 PM Bob doesn't exist as a B particle, particle 1 will not move with respect to the point occupied by Bob between 2 PM and 2:30 PM. .... and so on.

... If Bob changes into a B-particle he will never change back into an A particle ...each natural number is assigned to one assassin (Hawthorne [2000], pp. 628-9)

The sequence of time intervals he uses is disjoint, for the first assassin, 2 p.m. to 2.30 p.m., for the second 1.30 p.m. to 1.45 p.m., and so on. Call 2 p.m. to 2.30 p.m. the first time interval, 1.30 p.m. to 1.45 p.m. the second time interval, and so on. Our unbegun set is the ordinal $\omega$, with the 'before' relation being the reverse of the well order on $\omega$ (so 6 th is before 5 th, 5 th before 4 th, etc.). ${ }^{17}$ In general, the first conditional can be expressed as

1. if Bob is alive in the $n$th time interval he is killed by the $n$th Assassin, and the second conditional as

2. if Bob is not alive in the $n$th time interval he is not killed by the $n$th Assassin which together give us

3. Bob is killed by the $n$th Assassin iff he is alive in the $n$th time interval. We have

4. Bob is killed by the $n$th Assassin iff he is killed in the $n$th time interval. Hawthorne will perhaps object to the $R$ to $L$ direction, but this we get because if Bob is killed something kills him. In the $n$th time interval we can't appeal to a fusion of

${ }^{17}$ Strictly, if $<$ is the well order on $\omega$ and $k, m \in \omega$, then $k$ is before $m$ iff $m<k$. 
assassins to do the killing so it'll have to be the $n$th Assassin on pain of returning to uncaused events. 3 and 4 give us

5. Bob is alive in the $n$th time interval iff he is killed by the $n$th Assassin.

Because once dead Bob stays dead we have

6. Bob is alive in the $n$th time interval iff killed nowhere before the $n$th time interval

When 5 and 6 give us condition ANB

for all $n$ in S, Bob is killed in the $n$th time interval iff he is killed nowhere before the $n$th time interval.

So whilst Hawthorne seems to put forward a coherent possibility, the causal premiss that he needs for the sake of the point he wishes to make about the Change Principle proves to be his undoing. It is no help to him to restore coherence by abandoning that causal premiss. Abandoning it amounts to nothing more than specifying a set of sentences which can be satisfied by a possible world in which an infinitude of the $A$ particles sit where they sit, and a $B$ particle spontaneously changes into an $A$ particle at 1.00 p.m. Nothing wrong with that, but no interest in it either, and certainly no grounds on which to claim that the infinitude of A particles caused the change.

Angel

Angel offers ‘A Physical Model of Zeno’s Dichotomy’ within 'Newtonian collision mechanics'([2001], p. 347). We take the $x$ direction to be horizontal, the $y$ direction vertical, and ignore the $z$ direction. A spherical particle $M$ of radius 1 is travelling from left to right with velocity $(1,0)$. At $t_{0}$ its centre is at $(0,-1)$. A pair of countable infinities of particles of diminishing radii are arranged so that at time $t_{0}$ their centres are at $\left(-1 / 2^{n}, 1\right)$ and $\left(1 / 2^{n}, 1\right)$, for all $n \in \mathbb{N}$, each with velocity $\left(1,-2^{n}\right)$. ([2001], p. 350 section 3 and figure 1 on p. 352).

The horizontal velocities of $M$ and the other particles are identical, so we can treat the situation in terms of an inertial frame with velocity $(1,0)$ relative to the original inertial frame. In that frame $M$ is (initially, at least) stationary and the countable infinities of particles 'rain' down vertically. The radii of the countable infinities of particles have been chosen so that for all $n \in \mathbb{N}$, at time $t=1 / 2^{n}$ a pair of particles centred at $\left(-1 / 2^{n}, 0\right)$ and $\left(1 / 2^{n}, 0\right)$ with velocity $\left(0,-2^{n}\right)$ would contact $M$ if $M$ 's velocity remains unchanged prior to that time. ([2001], sentence crossing pp. 350-1). The particles all have the same mass.

Use of conservation of momentum and energy laws suffices to show that ... the particle pairs can never catch up with $M$ on the assumption that $M$ has had a collision with at least one higher indexed pair, which is required by our assumption being tested, namely that there is no earliest collision. (Angel [2001], p. 355)

The unbegun set, $\mathrm{S}$, is the set of times $\left\{t=1 / 2^{n}\right\}$. The condition ANB is for all $t$ in S, a pair of particles will collide with $M$ at time $t$ iff a pair of particles collide with $M$ at no time before time $t$. $L$ to $R$ holds because if it is hit at time $t$ it must have retained its initial velocity so not been hit before, and $R$ to $L$ holds because if it is not hit before, its initial velocity is unchanged so it is at the place to be hit by the pair of particles set up to hit it at that place at that time. 
Angel claims that his variant can be resolved by rejecting 'Principle $P$, the composition of contact interactions does not create a noncontact interaction'([2001], p. 349) (or by ruling out infinitudes of particles or placing upper bounds on velocities, which solutions are no of interest to us here).

Rejecting Principle $P$ would avoid my derivation of the $R$ to $L$ direction of ANB. However, I can re-write using 'interacted with' instead of 'hit', where interactions include non-contact interactions of this special sort. Now $L$ to $R$ of ANB holds because if particles interact with $M$ at time $t$ it must have remained with its initial velocity so not been interacted with before, and $R$ to $L$ holds because if it is not interacted with before, its initial velocity is unchanged so it is at the place to be interacted with by particles. Whence condition ANB, for all $t$ in S, particles interact with $M$ at time $t$ iff particles interact with $M$ at no time before $t$. Consequently we still have something contradictory when applied to $\mathrm{S}$ (because $\mathrm{S}$ is unbegun).

Angel claims that

even for some ontologically conservative physics at least as far as particle types and the Euclidean spacetime they inhabit are concerned, we must think of impact as a spatiotemporal phenomenon in which impact between $\mathrm{M}$ and a set of particles...may occur at $\mathrm{t}_{0}$ despite there being no spatial contact between $\mathrm{M}$ and the spatial limit of any subset of the...particles. ${ }^{18}$ 'Contact' in a deeper sense is not simply a spatial concept. ([2001], p. 357)

Angel could have made use of Hawthorne's rejection of the contact principle by appealing to the fusion of his particles, whose boundary includes the point $(0,0)$ at $t=$ 0 which contacts $M$. He doesn't want to, however, because that would undermine his desired rejection of Principle $P$. For that rejection, he needs it to be true that 'at $t_{0}$ there is [no] spatial contact between $\mathrm{M}$ and the spatial limit of any subset of the...particles'. But this is simply incorrect, and contact with the fusion of particles allows us to retain principle $P$.

The notion of 'contact in a deeper sense' seems to amount to the claim that rejecting principle $P$ introduces non-collision causation into collision mechanics. Principle $P$ is related to Hawthorne's Change Principle. Rejecting $P$ and asserting the existence of non-contact causal interactions would entail the falsity of the Change Principle, since we would have a case of changeless causation in which no change occurs to the set of raining particles but they cause a change in $M$. Rejecting $P$ is therefore objectionable for similar reasons to those I gave when discussing the Change Principle.

In claiming that rejection of Principle $P$ avoids the paradox Angel simply helps himself to the assumption that a non-contact interaction happens at a convenient time to avoid the contradiction (at $t=0$ ). Now of course, we have already proved that applying ANB to a begun set such as $S \cup\{0\}$ forces interaction at the first member. But the question is, what motivates adding zero to $\mathrm{S}$. We are not being offered well motivated constraints for the time and place of these non-contact interactions. As presented, the convenience is ad hoc.

Consequently rejecting principle $P$ leaves it undetermined what happens at time $t=$ 0 . There is an unbounded region in which $M$ cannot be found, but where it can be

${ }^{18}$ See footnote 15 again. 
found, in other words, what the effect of the non-standard impact of $\mathrm{S}$ on $M$ is, is undetermined. Indeterminism is not new in Newtonian mechanics, ${ }^{19}$ and what to do about it is much discussed. Earman ([1986], pp. 37-9) explains why he rejects some ways of avoiding it, and part of the significance of Perez Laraudogoitia's Beautiful Supertask ([1996]) is that it withstands many plausible evasions of indeterminacy. But in those cases which restrict themselves to collision mechanics without gravitation, the indeterminacy that arises is very different from that in Angel's case. The indeterminacy arises because of collisions, sometimes with time reversal, and whatever causation there is remains a matter of collision.

So rejecting $P$ does not amount to showing that it is possible within Newtonian collision mechanics for the raining particles to cause $M$ to escape to infinity without contacting $M$. It is, rather, a matter of renouncing causation in Newtonian collision mechanics and giving a set of sentences which can be satisfied by a possible world in which an infinitude of raining particles is accompanied by a particle which disappears, or in some other way instantaneously gets out of the way, at time zero. Angel is mistaking a logical constraint of satisfiability for a causal constraint. For these reasons I reject Angel's claim that 'we have a physical model for Zeno's dichotomy' (Angel [2001], p. 356). We have a model, but rejecting principle $P$ means it does not conform to Newtonian collision mechanics and is not a physical model of Zeno's dichotomy in any interesting sense of physical.

\section{Yablo and Sorensen}

Yablo’s paradox ([1993]) offers the infinite sequence of sentences, for $n \in \mathbb{N}$

$$
\mathrm{S}_{n} \text { : For all } k>n, \mathrm{~S}_{k} \text { is not true. }
$$

Here the unbegun set $S$ is $\mathbb{N}$, on which once again we take the 'before' relation to be the normal 'greater than' relation. Condition ANB is only implicit in the paradox, as a condition on the truth of the sentences. Let a sentence being true at $n$ be that $S_{n}$ is true. If a sentence is true at $n$, then since $S_{n}$ states that all sentence before it are not true, a sentence is true nowhere before $n$. Likewise, if a sentence is true nowhere before $n$, then for all $k$ before $n, S_{k}$ is not true, and so $S_{n}$, the sentence at $n$, is true. Thus condition ANB is that for all $n$ in $\mathbb{N}$, a sentence is true at $n$ iff a sentence is true nowhere before $n$.

In Sorensen's version of Yablo's paradox we have an infinite queue of students each of whom says 'Some of the students behind me are now thinking an untruth.' (Sorensen [1998], p. 137). The set $S$ is the queue, which is unbegun when we take 'behind' to be our 'before' relation. For convenience we can index the students in the queue with the natural numbers (with our now customary reverse ordering). Condition ANB is found in the condition on the students' thoughts being untrue. We take $E$ at $x$ to be the student at $x$ in the queue thinking an untruth. Plainly if the $n$th student thinking 'someone behind me is thinking an untruth' is thinking an untruth, then no one behind them is thinking an untruth $(\forall n$ (En $\rightarrow \neg \exists k(k<<n$ \& Ek)). Likewise, if noone behind the $n$th student is thinking an untruth then the $n$th student is thinking an untruth $(\forall n(\neg \exists k(k<<n \& E k) \rightarrow F n))$. So we have condition ANB: for all $n$ in $\mathbb{N}$, a

\footnotetext{
${ }^{19}$ See, for example, the discussion between Perez Laraudogoitia [1997] and Earman, J and Norton [1998].
} 
student is thinking an untruth at $n$ iff a student is thinking an untruth nowhere before $n$.

\section{Exploiting the form}

So in each of the variants of Benardete's dichotomy, and also in Yablo's paradox, we find the jointly unsatisfiable pair to be embedded. Why is this a solution? After all, in each case the initial conditions appear acceptable. I think the answer is that the appearance is something that has to be carefully managed, and since that management amounts to concealing the unsatisfiable pair, in presenting the dichotomies no possibility has been specified.

The mechanism of the dichotomy is to distribute the unsatisfiable pair over three inconsistent conditions, any pair of which is consistent:

1. An ordering on that set which makes it unbegun

2. A condition on an infinite set which is either

a) $\quad L$ to $R$ of Condition ANB or

b) A biconditional consisting of the LHS of Condition ANB and a RHS , $\Phi$.

3. A condition on an infinite set which is either

a) $\quad R$ to $L$ of Condition ANB or

b) A biconditional consisting of the RHS of Condition ANB and a LHS , $\Psi$.

The subtlety of the paradoxes is that either 2 or 3 is only implicit in the situation as described, and in the $(b)$ cases, that $\Phi \leftrightarrow \Psi$ is also implicit. The explicit situation offers two are jointly satisfiable conditions (either $1 \& 2$ or $1 \& 3$ ). Because the implicit condition is covert and complex, the three conditions appear to be independent and so bringing out the consequences of the implicit condition seems innocent. Consequently the paradoxical outcomes appear to come from satisfiable premisses.

Perez Laraudogoitia, Hawthorne, and Angel seek to strengthen the paradoxicality by rejecting the implicit principle whilst assuming a philosophically significant result remains. I think applying the unsatisfiable pair diagnosis shows that rejecting the implicit principle is done at the cost of the significant result.

We now turn to the matter of guile. Although the unsatisfiable pair are not obviously inconsistent, anyone presented bluntly with

Condition ANB: for all $x$ in S, E at $x$ iff $\mathrm{E}$ nowhere before

will find it easy to bring to mind its equivalent

Condition ANB*: for all $x$ in S, not $\mathrm{E}$ at $x$ iff $\mathrm{E}$ somewhere before $x$.

The conjunction of ANB and ANB* looks fishy, and the essence of lines 1 to 9 above is quickly seen:

$\mathrm{E}$ at $x$ iff E nowhere before $x$ (condition ANB)

iff for all $y$ before $x$, not $\mathrm{E}$ at $y$ (what ' $\mathrm{E}$ nowhere before $x$ ' means)

iff for all $y$ before $x$, E somewhere before $y$ (condition ANB* applied to the $y$ s before $x$, assumes unbegun condition)

only if E somewhere before $x$ (because the $y$ s are before $x$ ) 
only if not $\mathrm{E}$ at $x$ ( $R$ to $L$ of condition ANB* applied to $x$ )

Therefore Not $E$ at $x$. ( $P$ only if not- $P$ implies not- $P$ )

But if all that is quickly seen, the paradox will never get off the ground.

So distributing the unsatisfiable pair over three conditions is not just a matter of being less economical of expression, but necessary for the plausibility of the paradox. It is worth noting that Yablo ([2000]), wishing to refute Priest, gives a condition much closer to an explicitly stated condition ANB than anyone else.

Now that we know the form of the paradox we can make up new ones quite easily, and to illustrate further the matter of guile I exhibit an overt and a covert use of ANB.

Suppose a manufacturer claims to have invented a direct mode of transportation from A to any Z (not necessarily instantaneous). It is direct because, unlike normal transportation, arriving at $\mathrm{Z}$ is not a matter of passing through each of the intervening places between $A$ and $Z$. Their advert says 'we achieve this feat by arranging space in such a way that whenever there is a set of places on a path from A you don't arrive at, you arrive at the first place after it ${ }^{20}$ So directness means that if you arrive at $\mathrm{Z}$ then you didn't arrive anywhere before $Z$ and this is achieved by ensuring that if you didn't arrive anywhere before $\mathrm{Z}$ you will arrive at $\mathrm{Z}$. Since this is an example condition ANB, we now know that this machinery must either leave you at A or is logically impossible. But even if we didn't know that, the advertised condition lacks initial plausibility, and lacks it, I think because ANB is overt. We can immediately see that for any place Y before Z, it hasn't placed you at any of the points before that place Y, so you have to arrive at $\mathrm{Y}$ before $\mathrm{Z}$, not $\mathrm{Z}$, and this applies to all $\mathrm{Y}$ before $\mathrm{Z}$, so you just stay at A. ${ }^{21}$

With indirection we get something much more beguiling: consider a trolley, travelling from left to right on the $x$ axis, equipped with a flag on a small flagpole in its centre and a bad tempered elf who hates flags but is asleep. Along its path is a sequence of bad tempered goblins with bells who also hate flags. If the trolley reaches $1 / 2$ with its flag flying the goblin at that point will ring his bell and wake the elf who will lower the flag at 1 , if the trolley reaches $1 / 3$ with its flag flying the goblin at that point will ring his bell and wake the elf who will lower the flag at $1 / 2$, and in general if the trolley reaches $1 /(n+1)$ with its flag flying the goblin at that point will ring his bell and wake the elf who will lower the flag at $1 / n$. Then for all $x$ in $S=\{1 / n\}$, the flag will be no longer be flying at $x$ if and only if a goblin rang the bell before $x$. For every member of $\mathrm{S}$ there are two earlier members before it, at the first of which the goblin must have rung his bell if the flag was flying and at the second of which the elf will have lowered the flag. So for every member of $S$ the trolley will arrive with the flag down. But if that is true of every member of $S$, then no goblin saw a flag flying so no bell was rung so the elf remained sleeping and never lowered the flag so the flag must fly throughout.

The natural assumption by which a covert entanglement of ANB has been achieved is the assumption that unless lowered by the elf the flag will remain flying and the elf stays asleep unless woken by a bell. A bit of digging reveals the unsatisfiable pair. $\mathrm{S}$ is unbegun. Since for all $x$ in $S$, if the flag is lowered at $1 / n$ the goblin must have rung the bell at $1 /(n+1)$, so it wasn't lowered before $1 / n$. And if it wasn't lowered before

\footnotetext{
${ }^{20}$ To be strict, they mean that you arrive at the least upper bound of that set.

${ }^{21}$ But perhaps more plausible examples of overt entanglement could be created.
} 
$1 / n$ the goblin at $1 /(n+1)$ will ring the bell and the elf will lower the flag at $1 / n$. So for all $x$ in S, the flag will be lowered at $x$ if and only if it is lowered nowhere before $x$ (condition ANB). Despite appearances, then, we have failed to stipulate a real possibility. Inhabitants of this enchanted world will be spared an infinity of goblins each of whom both rings and doesn't ring his bell, an elf who is awake and asleep at the same time, and the chilling sight of a trolley passing by with its flag both up and down.

\section{Acknowledgements}

Many thanks to Michael Clark and to two anonymous referees for their helpful comments.

\section{Nicholas Shackel}

Department of Philosophy,

Kings' College

University of Aberdeen

Aberdeen

AB24 3UB

n.shackel@abdn.ac.uk

\section{References}

Angel, L. [2001]: 'A Physical Model of Zeno's Dichotomy’, British Journal for the Philosophy of Science, 52, pp. 347-58.

Benardete, J. [1964]: Infinity: An Essay in Metaphysics, Oxford: Clarendon Press.

Earman, J. [1986]: A Primer on Determinism, Dordecht: D. Reidel.

Earman, J. \& Norton, J. D. [1998]: 'Comments on Laraudogoitia's "Classical Particle Dynamics, Indeterminism and a Supertask"', British Journal for the Philosophy of Science, 49, pp. 123-33.

Hawthorne, J. [2000]: ‘Before Effect and Zeno Causality’, Noûs, 34, pp. 622-33.

Perez Laraudogoitia, J. [1996]: ‘A Beautiful Supertask’, Mind, 105, pp. 81.

Perez Laraudogoitia, J. [1997]: 'Classical Particle Dynamics, Indeterminism and a Supertask', British Journal for the Philosophy of Science, 48, pp. 49-54.

Perez Laraudogoitia, J. [2000]: 'Priest on the Paradox of the Gods', Analysis, 60, pp. 152-6.

Perez Laraudogoitia, J. [2003]: 'A Variant of Benardete's Paradox’, Analysis, 63, pp. 124-31.

Priest, G. [1999]: 'On a Version of One of Zeno's Paradoxes', Analysis, 59, pp. 1-2.

Sorensen, R. [1998]: 'Yablo's Paradox and Kindred Infinite Liars.', Mind, 107,

Sutherland, W. A. [1998]: Introduction to Metric and Topological Spaces, Oxford: Clarendon.

Yablo, S. [1993]: 'Paradox without Self-Reference', Analysis, 53, pp. 251-2.

Yablo, S. [2000]: ‘A Reply to New Zeno’, Analysis, 60, pp. 148-52. 